\title{
NOUVELLE
}

\section{Les bactériophages, alliés de l'épithélium intestinal contre les bactéries pathogènes}

Lorenzo Chaffringeon, Jholy De La Cruz, Valentin Dettling,

Elisa Eme-Scolan, Jéromine Samain

> Pour induire une infection intestinale, les bactéries pathogènes doivent surmonter plusieurs obstacles. En effet, différentes barrières de défense, regroupées sous le terme d'anté-immunité, entrent en jeu pour limiter le franchissement de la muqueuse intestinale par les bactéries. Ces barrières peuvent être mécaniques (comme les jonctions serrées entre cellules épithéliales), mais aussi chimiques (comme les grandes variations de $\mathrm{pH}$ le long du tractus digestif ou la sécrétion de lysozyme et autres composés antimicrobiens par les cellules de Paneth [1, 2]). Le mucus, qui contient des glycoprotéines telles que les mucines, accompagnées d'ADN et de débris cellulaires, forme un gel qui fonctionne comme une barrière supplémentaire [3].

Par ailleurs, une barrière écologique est assurée par les bactéries du microbiote résidant dans le tractus digestif, qui entrent en compétition avec d'autres populations bactériennes potentiellement pathogènes pour les ressources nutritives $[4,5]$. Les bactériophages interviennent également dans la régulation des populations bactériennes. Bien que ces virus de bactéries soient au moins aussi nombreux que les bactéries, leur rôle dans l'écosystème intestinal est encore peu exploré. Pour analyser plus précisément le rôle des bactériophages dans l'intestin, Barr et al. ont mené une étude fondée sur l'observation que, chez divers métazoaires (vertébrés et invertébrés), le nombre de phages rapporté au nombre de bactéries est significativement plus élevé dans le mucus que dans le lumen du tractus digestif [6].
Ces auteurs ont donc émis l'hypothèse que, du fait de son abondance en phages, le mucus pourrait être un lieu privilégié d'interaction entre phages et bactéries. Ils se sont dans un premier temps intéressés à l'adhérence du phage T4, virus de la famille des Myoviridae, et de l'ordre des Caudovirales s'attaquant à la bactérie Escherichia coli, à la surface de cellules produisant ou non du mucus. Pour cela, ils ont comparé in vitro le nombre de phages T4 adhérents à la surface d'hépatocytes (ne produisant pas de mucus), ou de cellules épithéliales de côlon (productrices de mucus). Ils ont constaté que le nombre de phages adhérents à la surface de cellules productrices de mucus était multiplié par deux, par rapport aux cellules n'en produisant pas. Les auteurs ont également observé une diminution d'adhérence des phages T4 à la surface de cellules épithéliales pulmonaires (productrices de mucus) traitées par un agent destructeur du mucus, par rapport à la même lignée cellulaire non traitée. Cela semble donc confirmer l'importance du mucus dans l'adhérence des phages à la surface cellulaire.

Ensuite, afin de mettre en évidence les composants du mucus essentiels à l'adhérence des phages, les auteurs ont évalué l'adhérence des phages T4 sur des plaques d'agar recouvertes de différents composants du mucus. Ils ont observé que la présence de mucines à la surface de plaques d'agar augmentait significativement le nombre de phages adhérents. II semblerait donc que les mucines soient un élément important pour l'adhérence des phages au mucus.
École normale supérieure de Lyon, département de biologie, Master biologie, Lyon, France.

Les auteurs ont ensuite cherché à déterminer les éléments structuraux permettant au phage T4 d'adhérer au mucus. Comme tous les phages appartenant à l'ordre des Caudovirales ou phages dits « à queue », ce phage est composé de trois grandes parties: la capside qui contient et protège le génome d'ADN double brin, la queue qui permet l'injection de I'ADN dans la bactérie infectée et les fibres caudales nécessaires à l'attachement du phage T4 à la paroi bactérienne. La capside du phage T4 est constituée, entre autres, de la protéine Hoc dont la structure présente quatre domaines dits Ig-like correspondant à un repliement de type immunoglobuline, constitué de brins $\beta$ associés en deux feuillets $\beta$ [7]. Les brins $\beta$ sont séparés par des boucles conférant à la structure une certaine flexibilité. Les auteurs ont émis l'hypothèse que la flexibilité des domaines lg-like de la protéine Hoc permet la reconnaissance et l'adhérence des phages au mucus. II s'est avéré que des phages T4 sauvages adhèrent significativement plus que des phages T4 déficients pour la protéine $\mathrm{Hoc}$, indiquant que la protéine $\mathrm{Hoc} d u$ phage T4 est nécessaire à l'adhérence au mucus.

D'autre part, afin de tester la spécificité d'interaction entre les phages et les mucines, les auteurs ont évalué l'adhérence de phages sur une puce présentant 610 résidus glycanes distincts. Ces expériences ont permis de mettre en évidence une spécificité d'interaction entre la protéine $\mathrm{Hoc}$ et des glycanes dont certains sont spécifiques des mucines. 


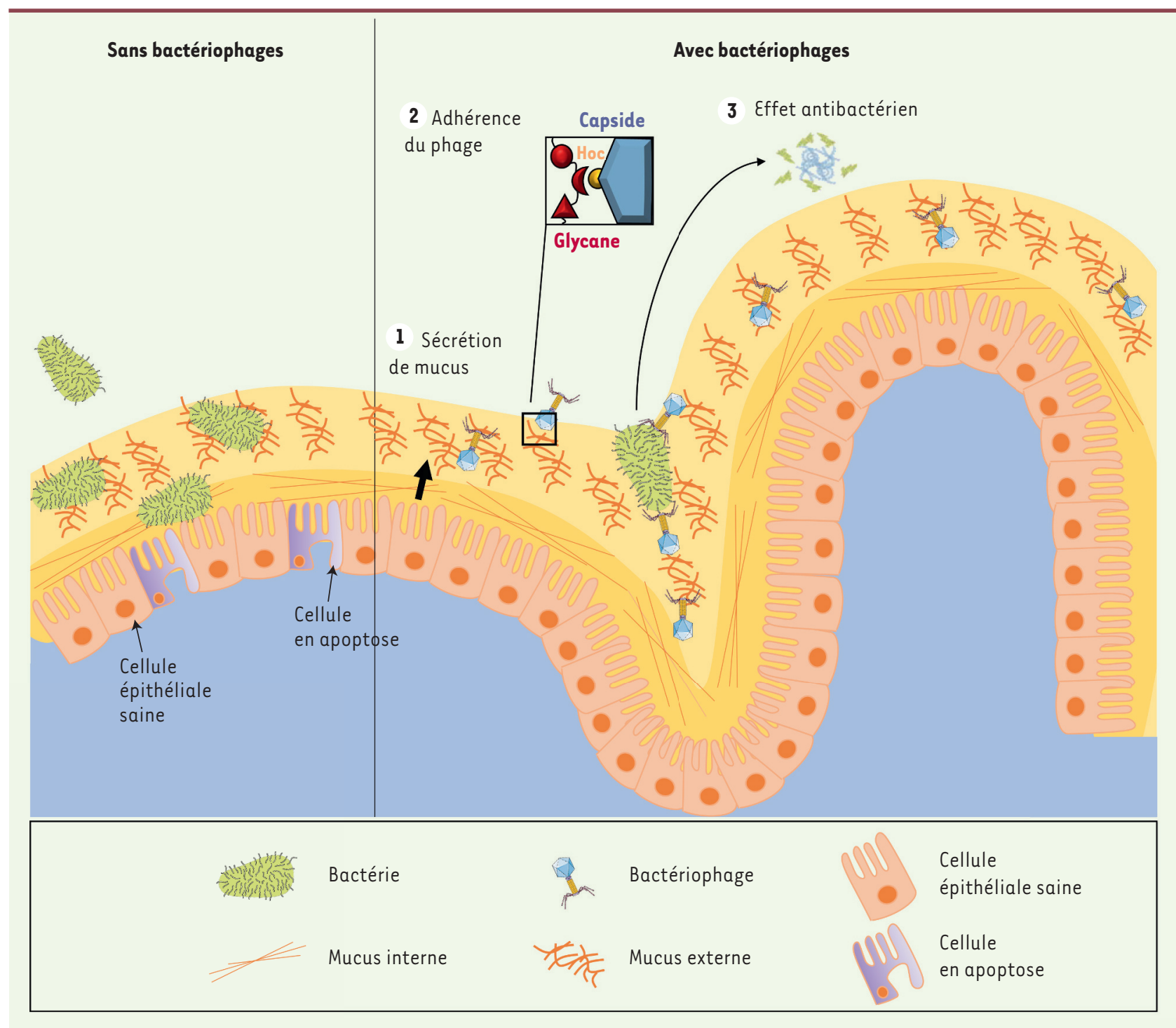

Figure 1. Le modèle « bacteriophage adherence to mucus » (BAM). Comparaison d'un épithélium intestinal avec ou sans bactériophages, mettant en évidence leur rôle essentiel dans le maintien de l'homéostasie intestinale. 1. Le mucus est sécrété par l'épithélium intestinal sous-jacent. 2. Les bactériophages T4 se lient aux glycanes des mucines via les domaines lg-like de leur protéine de capside Hoc. 3. L'adhérence des phages permet de constituer une couche à activité antibactérienne et donc un niveau de défense supplémentaire.

Afin de déterminer si cette interaction entre phages et mucus pouvait avoir un rôle fonctionnel, notamment sur l'adhérence bactérienne aux cellules épithéliales, les auteurs ont réalisé une co-culture phage T4 - bactérie $\varepsilon$. coli cellules humaines épithéliales productrices de mucus. Ils ont ainsi observé qu'en présence de phages, la capacité d'adhérence des bactéries était considérablement réduite. Pour déterminer le mécanisme expliquant cette réduction d'attachement bactérien, les auteurs ont utilisé une stratégie expérimentale originale. Ils ont en effet de nouveau réalisé l'expérience précédente, une co-culture phage-bactérie-cellules humaines, mais en utilisant un phage mutant qui n'a pas la capacité de se répliquer, et ne peut donc réaliser qu'un seul cycle d'infection. Dans ces conditions, la diminution de l'adhérence des bactéries en présence des phages est dépendante de la capacité de réplica- tion de ces derniers. Ceci suggère donc que c'est grâce à une lyse bactérienne, résultat de la réplication virale, que les phages réduisent l'attachement bactérien.

L'adhérence des phages au mucus leur permet donc de réduire de manière active, par lyse bactérienne, l'attachement des bactéries aux cellules eucaryotes. Cela suggère un effet protecteur des cellules épithéliales par les phages, contre les bactéries pathogènes. Afin de 
vérifier cette hypothèse, les auteurs ont étudié la mortalité de cellules épithéliales humaines en co-culture avec $\varepsilon$. coli et/ou des phages T4. Ils ont observé qu'en présence de phages T4, la mortalité cellulaire induite suite à l'infection par $\varepsilon$. coli est diminuée. Cet effet n'est plus observé lorsque les cellules sont déficientes pour la production de mucus, confirmant donc que les phages, en adhérant au mucus et en induisant la lyse des bactéries, protègent les cellules épithéliales des effets pathogènes de l'infection bactérienne.

Cette étude permet donc d'émettre l'hypothèse d'une synergie fonctionnelle entre le mucus du tractus intestinal et les phages présents dans celui-ci, définissant le modèle bacteriophage adherence to mucus (BAM) comme un niveau supplémentaire de protection antibactérienne assurée par le mucus, au-delà de la barrière physico-chimique déjà décrite dans la littérature (Figure 1). Cette étude, réalisée in vitro, devra être complétée par d'autres travaux afin de conclure sur la pertinence de ces interactions phages/mucus in vivo. Le modèle BAM pourrait par exemple être affiné en considérant les différents types de mucus et leur distribution in vivo. En effet, deux types principaux de mucus sont décrits dans le côlon humain: une couche de mucus externe épaisse directement en contact avec le microbiote, et une couche interne mince en contact avec l'épithélium. Ces deux couches diffèrent notamment par la solubilité du réseau de mucines, qui gouverne leur vitesse de dégradation mais aussi par l'exposition des glycanes qui leur sont associés $[8,9]$. La couche interne serait ainsi plus stable que la couche externe, soumise, elle, à une dégradation plus importante. Cette dégradation permet de rendre les glycanes de la couche externe plus accessibles, ce qui se traduit par une probabilité d'interaction plus élevée entre les phages et les mucines par rapport à la couche interne du mucus.

Par ailleurs, le modèle BAM pourrait être intégré à d'autres aspects des relations phages/bactéries. En effet, il existe chez les mammifères une diversité très importante de phages spécifiques non seulement de certains pathogènes, mais aussi de bactéries commensales du microbiote. La relation proie-prédateur entre phages et bactéries permet de contrôler les populations bactériennes et empêche la prédominance d'une espèce bactérienne qui pourrait perturber la diversité du microbiote. Cela suggère donc non seulement une fonction anti-pathogène du BAM, mais aussi une fonction de régulation du microbiote de l'hôte.

Dans cette étude, le bactériophage utilisé comme modèle est le phage T4, qui suit un cycle lytique. Les phages tempérés, c'est-à-dire à cycle lysogénique, dont le génome est capable de s'intégrer dans le génome bactérien, pourraient également avoir un rôle au cours de pathologies inflammatoires. En effet, certaines bactéries du microbiote possèdent dans leur génome des prophages qui ont la capacité d'entrer en cycle lytique en cas de stress inflammatoire, pouvant ainsi entraîner une dysbiose, c'est-à-dire un déséquilibre dans la composition des populations bactériennes du microbiote [10]. $\varepsilon$ n conclusion, cette étude apporte de nouveaux éléments dans le domaine des relations symbiotiques entre les virus et les métazoaires. En mettant en évidence les détails moléculaires de l'interaction hôte/phage et ses conséquences dans la lutte antibactérienne, elle pourrait constituer un pilier dans le développement de la phagothérapie. $\diamond$

The key role of bacteriophages in the defense of the gut epithelium against pathogenic bacteria

\section{LIENS D'INTÉRÊT}

Les auteurs déclarent n'avoir aucun lien d'intérêt concernant les données publiées dans cet article.

\section{RÉFÉRENCES}

1. Viggiano D, Ianiro G, Vanella G, et al. Gut barrier in health and disease: focus on childhood. Eur Rev Med Pharmacol Sci 2015 ; 19 : 1077-85.

2. Riera Romo M, Pérez-Martínez D, Castillo Ferrer C. Innate immunity in vertebrates: an overview. Immunology 2016 ; 148 : 125-39.

3. Cornick S, Tawiah A, Chadee K. Roles and regulation of the mucus barrier in the gut. Tissue Barriers 2015 ; 3 : e982426.

4. Stecher $B$. The roles of inflammation, nutrient availability and the commensal microbiota in enteric pathogen infection. Microbiol Spectr 2015 ; 3. doi: 10.1128/microbiolspec.

5. Kayama H, Takeda K. Functions of innate immune cells and commensal bacteria in gut homeostasis. J Biochem (Tokyo) 2016; 159 : 141-9.

6. Barr JJ, Auro R, Furlan M, et al. Bacteriophage adhering to mucus provide a non-host-derived immunity. Proc Natl Acad Sci USA 2013 ; 110 : 10771-6.

7. Bork P, Holm L, Sander C. The immunoglobulin fold. Structural classification, sequence patterns and common core. J Mol Biol $1994 ; 242$ : 309-20.

8. Johansson MEV, Larsson JMH, Hansson GC. The two mucus layers of colon are organized by the MUC2 mucin, whereas the outer layer is a legislator of hostmicrobial interactions. Proc Natl Acad Sci USA 2011 ; 108 (suppl 1) : 4659-65.

9. Li H, Limenitakis JP, Fuhrer T, et al. The outer mucus layer hosts a distinct intestinal microbial niche. Nat Commun $2015 ; 6: 8292$.

10. Duerkop BA. Bacteriophages shift the focus of the mammalian microbiota. PLoS Pathog 2018 ; 14 : e1007310.

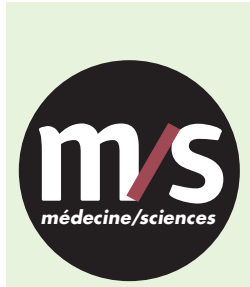

Tarifs d'abonnement m/s - 2019
Abonnez-vous
à médecine/sciences
$>$ Grâce à $\mathrm{m} / \mathrm{s}$, vivez en direct les progrès des sciences biologiques et médicales

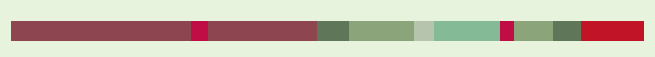

$$
\begin{gathered}
\text { Bulletin d'abonnement } \\
\text { page } 594 \text { dans ce numéro de } \mathrm{m} / \mathrm{s}
\end{gathered}
$$

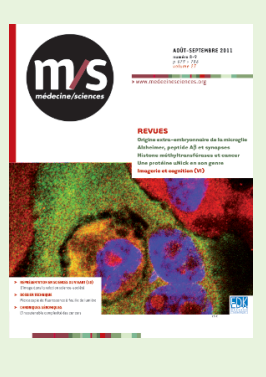

